\title{
Fulminant Hepatic Failure Resulting from Coexistent Wilson's Disease, Hepatitis E and Herbal Medication: A Case Report
}

\author{
Baliga Kiran*, Pradyum Ram, Baliga BS , Rajesh SM and Kini Prashant
}

Department of Pediatrics, KMC Mangalore, Manipal University, India

\begin{abstract}
Infection with hepatitis A virus can cause severe or even fatal illness in patients with chronic liver disease. Here we describe a ten-year-old girl who presented as acute liver failure and was diagnosed with Wilson's disease and later with coexistent hepatitis E infection. Wilson's disease was demonstrated on the basis of Kayser Fischer ring, low ceruloplasmin, and high urinary copper excretion. Hepatitis E was diagnosed serologically with a positive anti HEV IgM titers. Our case suggests that acute hepatitis E may play a part in the acute decompensation seen in some cases of unrecognized Wilson's disease. The patient gradually deteriorated and died in a period of 3 months.
\end{abstract}

Keywords: Acute liver failure; Hepatitis E infection; Wilson's disease

\section{Introduction}

Fulminant Wilson's disease is characterized by severe derangement of liver function, encephalopathy, and hemolysis in patients with previously undiagnosed liver disease. Early diagnosis is essential as without orthoptic liver transplantation, the mortality is virtually $100 \%$. Although the basic defect, a failure of biliary copper excretion, has been known for many years, the exact pathophysiology of the defective excretory mechanism has remained elusive. It is known that Wilson's disease may run a varied course, ranging from fulminant Wilson's disease to an insidious progression to cirrhosis over 20-30 years. The factors that dictate disease tempo in any particular patient remain unknown. Many patients with fulminant Wilson's disease have grossly raised serum copper concentrations, and it has been postulated that an acute insult damages the hepatocytes, which release free copper in toxic concentrations, which in turn damage cell membranes both directly [1] and by free radical mediated damage ${ }^{2}$. It is therefore possible that in some patients a hepatic injury unrelated to the metabolic abnormalities of Wilson's disease in it may be the primary process that triggers fulminant Wilson's disease. This study reports on a case of fulminant hepatic failure occurring in a ten year old girl with biochemical evidence for underlying Wilson's disease complicated by Hepatitis E Virus (HEV) infection. The diagnosis of HEV was made by nested polymerase chain reaction and confirmatory DNA sequencing. The patient required emergency orthotopic liver transplantation but due to financial constraints was unable to afford the same. The histopathology of HEV, the time course of viral shedding in stool, the viral titer in serum, and the relation to Wilson's disease are discussed.

\section{Case Report}

Our patient 10 years female was referred by a Pediatrician with history of fever since 1 day following which immediately developed jaundice. History of alternative medicine was present (herbal medicine). Jaundice deepened on day 3 when she was seen by the pediatrician where she was investigated for the same. Liver Function Tests (LFT) where done where Totals Bilirubin (TB) came to be $7.8 \mathrm{mg} / \mathrm{dl}$ with no evidence of hepato-trophism. Within 24 hours of the previous LFT, $\mathrm{TB}$ results came to be $23 \mathrm{mg} / \mathrm{dl}$ and she developed acute fulminant hepatitis. On admission initial diagnosis of toxin induce hepatitis or unconventional hepatitis was made.

At the time of admission the investigations done on the patient revealed the following results. Hemoglobin was $7.6 \mathrm{~g} / \mathrm{dl}$. Peripheral smear revealed Normocytic normochromic polychromasia and 1 normal RBC/100 WBC, reticulocyte count 14, s/o haemolytic anaemia. Electrolytes being Sodium 132; Potassium 4.4;CHloride 106; Bicarbonates- 17.5;Calcium-8.8.TB $37.2 \mathrm{mg} / \mathrm{dl}$; DB $26.4 \mathrm{mg} /$ dl; SGPT $32 \mathrm{U} / \mathrm{L}$; SGOT $212 \mathrm{U} / \mathrm{L}$; ALP $32 \mathrm{U} / \mathrm{L}$; Total protein $6.9 \mathrm{~g} /$ dl; Albumin $2.8 \mathrm{~g} / \mathrm{dl}$; PT (Test) 32.3s (Control) 13s; INR 2.68; AntiHEV Ig M positive; Other Viral markers Negative; RFT- normal study; serum ceruloplasmin - $13.7 \mathrm{mg} / \mathrm{dl}$ (20-60); serum ferritin $1761 \mathrm{ng} /$ $\mathrm{ml}$ (13-150); $24 \mathrm{hr}$ urine copper 3310; serum copper 48. Toxic screen Permissible limits of heavy metals in herbal medicine - Lead $10 \mathrm{ppm}$, Cadmium 0.30 ppm, Arsenic 10 ppm, Mercury 1 ppm.

Liver biopsy could not be performed due to severe coagulopathy. Ophthalmology opinion showed early KF ring present in both eyes inferiorly and superiorly. The child's parents were advised to shift the child to higher centre for liver Transplantation. Details of procedure along with financial implications, donor requirement the risks involved were explained along with maximum survival of around 6 months without the transplant were explained to parents, however the parents expressed inability to meet the cost. The siblings were screened for Wilson's disease.

At the time of discharge TB- 16.4, DB - 9.2, TP- 7.7, Alb - 3.0, Glob- 4.7, AST- 320, ALT- 180, ALP - 139, PT-73.3(T), C- 13.0, INRnot possible. The patient gradually deteriorated over 3 months and died [2].

\section{Discussion}

It is probable that the sudden deterioration of her liver function leading to hepatic failure was brought about by the superimposed acute hepatitis E. Fulminant hepatic failure resulting from hepatitis E, well recognized during 'pregnancy and in neonates' $[3,4]$ is, however, distinctly rare in children. It is possible that the severity of the liver damage in this case derives from the coincidental presence of two rare conditions. The acute viral insult superimposed to the underlying Wilson's disease may have caused release of free copper in sufficient concentrations to provoke hemolysis and progressively more

*Corresponding author: Kiran N Baliga, Department of Pediatrics, KMC, Mangalore, India, Tel: 0824242 2271; E-mail: baligakiran@gmail.com

Received February 19, 2015; Accepted April 19, 2015; Published April 22, 2015

Citation: Kiran B, Ram P, Baliga BS, Rajesh SM, Prashant K (2015) Fulminant Hepatic Failure Resulting from Coexistent Wilson's Disease, Hepatitis E and Herbal Medication: A Case Report. J Clin Case Rep 5: 526. doi:10.4172/21657920.1000526

Copyright: ( 2015 Kiran B, et al. This is an open-access article distributed under the terms of the Creative Commons Attribution License, which permits unrestricted use, distribution, and reproduction in any medium, provided the original author and source are credited. 
severe liver damage. It is also possible that the pre-existing Wilson's disease may have predisposed the girl, if not to viral infection, to an exacerbation of its consequences. It is conceivable that viral infections, particularly those producing hepatitis, may be responsible, at least in some cases, for the acute decompensation seen in fulminant Wilson's disease. Diagnosis of Wilson's disease in the context of fulminant hepatic failure is notoriously difficult because abnormal serum copper concentration, high urinary copper excretion, and low ceruloplasmin concentrations are common in acute liver failure not caused by Wilson's disease. Typically, though, these patients have low liver copper content, because of severe liver cell necrosis. In the absence of chronic cholestatic liver disease or high oral copper intake, abnormal biochemical copper studies in conjunction with histopathological evidence of excess copper and copper associated protein in a non-cirrhotic liver are diagnostic of homozygous Wilson's disease in its early stages. In our case, the 24 hour urinary copper excretions before and after penicillamine challenge were at the values seen in Wilson's disease and the liver copper content was 3-15 times the upper limit of normal, well within the range seen in fulminant Wilson's disease. As recently described in the course of acute hepatitis E transmitted to a volunteer, our patient had detectable HEV RNA both in serum and stools at the time of acute symptomatic hepatitis.

Our patient presented like ALF with no previous history of liver disease. After completing the laboratory work-up for determining the etiology of liver failure, we came to the conclusion that the disease was hepatitis E super infection on a liver already affected by WD. Symptoms of WD are nonspecific and are associated with persisting asymptomatic elevation of aminotransferase concentrations, progressive development of cirrhosis, and fulminant liver failure. In a patient presenting with ALF, fulminant WD should also be considered in the differential diagnosis $[5,6]$.

Fulminant WD is characterized by severe derangement of liver function, encephalopathy, and hemolysis in patients with previously undiagnosed liver disease. Presence of very high serum copper levels, Coombs negative hemolytic anemia, low alkaline phosphatase levels despite high bilirubin levels, and relatively low elevations of aminotransferases, and higher AST than ALT levels should alert the physician to the diagnosis of fulminant WD. However, diagnosis of WD in a patient presenting with ALF is a diagnostic challenge because abnormal serum copper concentration, high urinary copper excretion, and low ceruloplasmin concentrations are common in ALF cases secondary to the causes of liver failure other than WD. Although liver biopsy is dangerous in patients with ALF because of clotting problems, high hepatic copper content is crucial for proper diagnosis. Early diagnosis is essential since without orthotropic liver transplantation, the mortality is virtually $100 \%$. Histologic examination of explanted livers of patients who underwent liver transplantation due to fulminant WD usually demonstrated established cirrhosis instead of massive necrosis. Our patient had established liver cirrhosis due to WD. She had marginally low ceruloplasmin, and raised serum, urine, and hepatic copper levels. The commonly used clinical and laboratory parameters were not sufficient to exclude the diagnosis of WD in patients with liver disease of unknown origin. In 2003, Ferenci et al. 12 developed a scoring system for diagnosing WD. According to the Ferenci score, our patient had a score of 5, indicating diagnosis of WD was highly probable. It was reported that ALF with WD differed from idiopathic ALF by higher copper levels in serum, urine and liver, less pronounced elevations of transaminase levels, higher concentrations of serum bilirubin, and lower hemoglobin values11.

Sallie et al. [5] reported a case of fulminant liver failure resulting from coexisting WD and hepatitis E. This is the only article in the English literature that described a child with underlying WD who developed ALF precipitated by a viral infection. This patient had Coombs negative hemolytic anemia, and blood and urine copper studies were consistent with the diagnosis of WD. Massively raised liver copper stores with regional variation (mean $445 \mu \mathrm{g} / \mathrm{g} / \mathrm{dry}$ weight) were determined in the resected liver during liver transplantation. Histopathologically there was extensive cell loss and mixed inflammatory cell infiltration, with no evidence of cirrhotic transformation. The authors reported that the acute viral insult superimposed on the underlying WD might have caused release of free copper and provoked hemolysis and severe liver damage

\section{Conclusion}

In conclusion, this study clearly shows that acute HEV infection in patients with cirrhosis produces decompensation of underlying liver disease and is associated with a high mortality rate. This case suggests that detectable or undetectable viral infections may play a part in fulminant presentation seen in some cases of WD.

\section{References}

1. Aaseth J, Ribarov S, Bochev P (1987) The interaction of copper (Cu++) with the erythrocyte membrane and 2,3-dimercaptopropanesulphonate in vitro: a source of activated oxygen species. Pharmacol Toxicol 61: 250-253.

2. Fernandes A, Mira ML, Azevedo MS, Manso C (1988) Mechanisms of hemolysis induced by copper. Free Radic Res Commun 4: 291-298.

3. Bradley DW (1990) Enterically-transmitted non-A, non-B hepatitis. Br Med Bull 46: 442-461.

4. Song DY, Zhuang H, Kang XC (1991) Hepatitis E in Hetian City: a report of 562 cases. Viral hepatitis and liver disease. Williams \& Wilkins Baltimore, USA.

5. Martins da Costa C, Baldwin D, Portmann B, Lolin Y, Mowat AP, et al. (1994) Value of urinary copper excretion after penicillamine challenge in the diagnosis of Wilson's disease. Hepatology 15: 609-615.

6. R Sallie, J Chiyende, Tan KC, D Bradley, B Portmann (1994) Fulminant hepatic failure resulting from coexistent Wilson's disease and hepatitis E. Gut 35: 849853. 